\section{Carbamylation des protéines}

\section{Quand vieillissements protéique et chronologique se rejoignent}

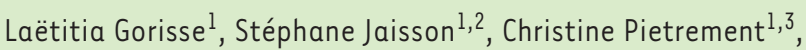
Philippe Gillery ${ }^{1,2}$
'Université de Reims Champagne-Ardenne, CNRS UMR 7369 , Laboratoire de biochimie médicale et biologie moléculaire, UFR de Médecine, 51, rue Cognacq-Jay, 51095 Reims Cedex, France ;

${ }^{2} \mathrm{CHU}$ de Reims, Pôle de biologie médicale et pathologie, Laboratoire de biologie et de recherche pédiatriques, 45, rue Cognacq-Jay, 51092 Reims Cedex, France ; ${ }^{3} \mathrm{CHU}$ de Reims, American memorial hospital, Unité de néphrologie pédiatrique, 47, rue Cognacq-Jay, 51092 Reims Cedex, France. pgillery@chu-reims.fr
> Les mécanismes moléculaires associés au vieillissement chronologique font encore l'objet de nombreuses hypothèses. Une littérature abondante a démontré l'implication, à des degrés divers, de la sénescence cellulaire, du raccourcissement des télomères, de la diminution de la capacité proliférative des cellules, des mutations de I'ADN mitochondrial, ou encore de facteurs inflammatoires dans le vieillissement chronologique. Cependant, un phénomène rarement évoqué est le vieillissement protéique, caractérisé par l'altération des propriétés structurales et fonctionnelles des protéines liée à des modifications post-traductionnelles non enzymatiques (MPTNE) [1]. Nous avons récemment montré que l'une d'entre elles, la carbamylation, était directement liée au vieillissement chronologique et à la longévité dans trois espèces animales [2].

\section{Vieillissement des protéines :} un phénomène naturel et délétère Tout au long de leur séjour dans l'organisme, les protéines sont la cible de MPTNE (Figure $1 \mathrm{~A}$ ), des réactions chimiques qui ne sont pas régulées et qui surviennent de façon naturelle mais inéluctable. Ces réactions sont connues depuis plus d'un siècle dans l'industrie alimentaire. L'une d'elles, la glycation non enzymatique, qui correspond à la condensation de sucres sur des protéines, est décrite sous le nom de réaction de Maillard [3]. On a pendant longtemps pensé que cette réaction ne pouvait pas se dérouler in vivo, même avec moins d'intensité, jusqu'à ce que sa survenue soit démontrée au cours du diabète sucré et, à un niveau basal, dans les conditions physiologiques [1]. Il y a vingt ans, l'équipe de Vincent Monnier a mis en évidence, dans différentes espèces, une accumulation des produits de glycoxydation (glycation non enzymatique associée à des réactions oxydatives) dans la peau au cours du vieillissement [4]. Nos récents résultats démontrent un lien direct entre la carbamylation, une autre MPTNE, et le vieillissement de l'organisme [2], les protéines étant d'autant plus susceptibles d'être carbamylées que leur durée de vie est longue.

\section{Carbamylation : une histoire ancienne mais méconnue}

La carbamylation correspond à la fixation irréversible et cumulative d'acide isocyanique (de formule $\mathrm{HN}=\mathrm{C}=0$ ) sur les groupements aminés des protéines, en particulier sur les résidus de lysine, pour former de l'homocitrulline (Figure IB). Dans l'organisme, l'acide isocyanique résulte principalement de la dissociation spontanée de l'urée, ce qui explique que l'intensité de la carbamylation soit augmentée au cours de l'insuffisance rénale, caractérisée par une élévation de l'urémie. Il peut également être formé de façon enzymatique par l'action de la myéloperoxydase sur le thiocyanate, en particulier au niveau des plaques d'athérosclérose. Enfin, un apport atmosphérique a été décrit à partir de la combustion de la biomasse, mais cette source exogène paraît très mineure [5]. L'importance de la réaction de carbamylation en physiopathologie est évoquée depuis une quarantaine d'années. À cette époque, l'urée et le cyanate étaient utilisés à des fins thérapeutiques afin de diminuer la précipitation de l'hémoglobine $S$ chez les patients atteints de drépanocytose ${ }^{l}$. La carbamylation entraîne en effet une diminution de l'agrégabilité de cette hémoglobine mutée et une augmentation de son affinité pour l'oxygène. Cependant, un effet secondaire inattendu était le développement de cataracte, provoquée par la carbamylation des protéines du cristallin [6]. Pendant longtemps, la carbamylation a été négligée par rapport à la glycation, essentiellement parce que les méthodes d'investigation biologique étaient prises en défaut. Désormais, les techniques de chromatographie en phase liquide couplée à la spectrométrie de masse en tandem (LC-MS/MS) permettent un dosage fiable et sensible des produits de carbamylation. En 2013, nous avons montré pour la première fois que les produits de carbamylation s'accumulaient dans l'organisme d'animaux atteints d'insuffisance rénale chronique [7]. Cette observation fournissait un substratum physiopathologique aux études cliniques récentes montrant que la carbamylation des protéines sériques était associée à la morbi-mortalité chez les patients atteints de troubles rénaux ou cardiaques [8]. La carbamylation affecte les propriétés physico-chimiques des protéines et leurs

\footnotetext{
${ }^{1}$ La drépanocytose, aussi appelée anémie falciforme, est une maladie héréditaire touchant l'hémoglobine. L'hémoglobine mutée des patients drépanocytaires (dite hémoglobine S pour Sickle, faucille en anglais) a tendance à polymériser lorsque la concentration d'oxygène dans le sang est faible. Les polymères d'hémoglobine $S$ déforment alors les hématies et leur donnent une forme caractéristique de faucille.
} 
A

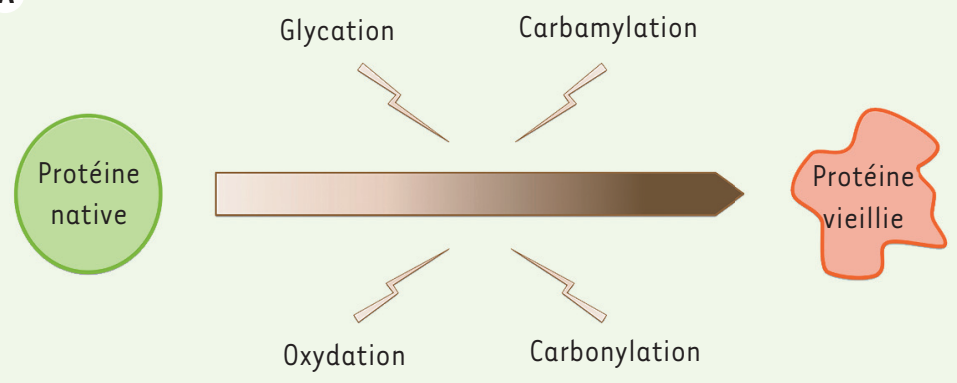

B

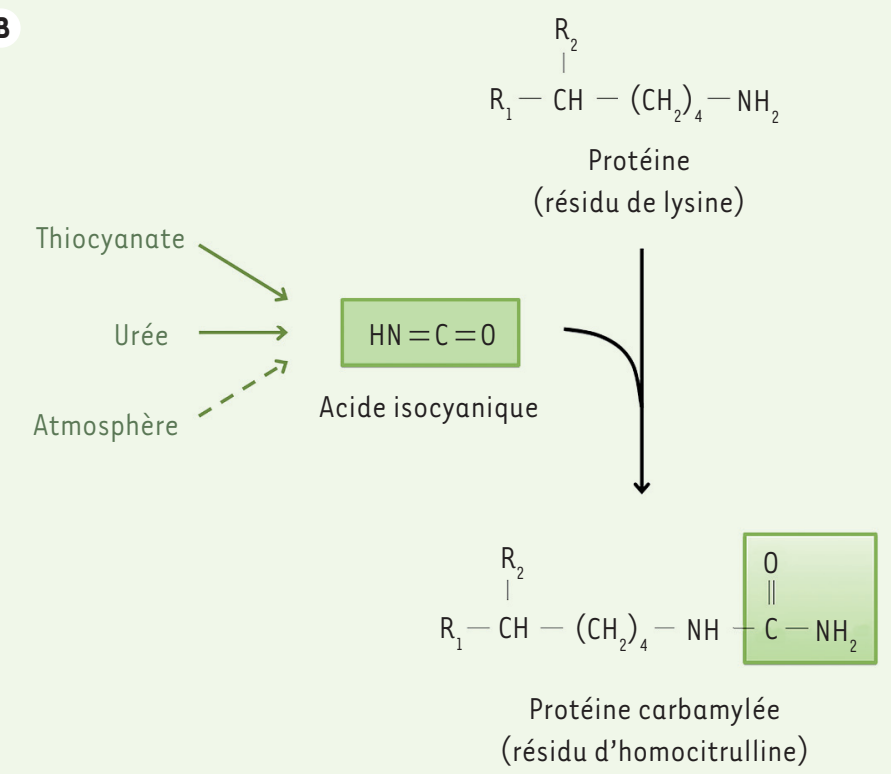

Figure 1. Le vieillissement protéique. A. Les modifications post-traductionnelles non enzymatiques des protéines. $\boldsymbol{B}$. La réaction de carbamylation.

interactions avec les cellules. Ainsi, le collagène de type I et/ou l'albumine carbamylée provoquent des réactions inappropriées des monocytes et des polynucléaires neutrophiles, pouvant expliquer les troubles inflammatoires ou infectieux rencontrés au cours de l'insuffisance rénale chronique $[9,10]$.

\section{Carbamylation et vieillissement : \\ un nouveau concept}

L'une des questions, restées en suspens, était l'éventualité d'une accumulation des produits de carbamylation dans l'organisme au cours du vieillissement. Nous avions déjà observé que le taux basal de carbamylation des protéines augmentait chez l'animal de laboratoire au cours d'expériences à relativement court terme. Pour répondre à la question, nous avons évalué la carbamylation des protéines cutanées, par dosage de l'homocitrulline par LC-MS/MS, dans trois espèces de mammifères ayant des durées de vie très différentes : la souris (avec des prélèvements de la naissance jusqu'à deux ans), le bœuf (avec des prélèvements de six mois à huit ans, la durée de vie du bøuf étant d'environ vingt ans) et l'homme (avec des prélèvements entre un mois et quatre-vingt-dix-huit ans). Nous avons étudié la carbamylation, en particulier du collagène de type I et de l'élastine, des protéines matricielles majeures dont la durée de vie longue en fait des cibles préférentielles (Figure 2A). Les résultats que nous avons obtenus ont montré qu'il existait, en fonction de l'âge, une augmentation progressive de la carbamylation des protéines cutanées, en particulier du collagène, dans les trois espèces étudiées. Les taux de carbamylation étaient deux à trois fois plus élevés chez le bœuf et l'homme que chez la souris aux âges les plus avancés, ce qui démontrait une accumulation progressive d'autant plus importante que la durée de vie du sujet est longue (Figure 2B). De façon intéressante, cette accumulation se produit à une vitesse différente selon les espèces. Elle est d'autant plus rapide que la durée de vie de l'espèce est courte et que la concentration basale d'urée est élevée. Une corrélation inverse a ainsi clairement été observée entre l'espérance de vie moyenne et la vitesse d'augmentation de la carbamylation. De façon inattendue, l'intensité de la carbamylation mesurée lors de ces expériences était supérieure à celle détectée pour la glycoxydation, les valeurs d'homocitrulline étant près de trois fois plus élevées que celles de la carboxyméthyllysine, considérée comme un produit majeur de glycoxydation tissulaire. Au vu de ces résultats, nous avons cherché à savoir si l'accumulation tissulaire de ces produits de carbamylation pouvait être réversible. Nous avons donc induit la carbamylation par supplémentation de cyanate de sodium dans l'eau de boisson chez la souris et avons observé que les protéines carbamylées tissulaires n'étaient en fait qu'en partie renouvelées, leur turnover paraissant insuffisant pour que l'organisme s'en débarrasse totalement.

\section{Vers de nouvelles stratégies?}

Les questions posées par nos observations nécessitent des approches expérimentales complémentaires. II convient en particulier de mieux préciser les liens entre carbamylation des protéines et pathologies. II est également nécessaire d'identifier les éventuels mécanismes de protection contre la carbamylation, comme l'existence d'enzymes de décarbamylation. Ainsi, la meil- 


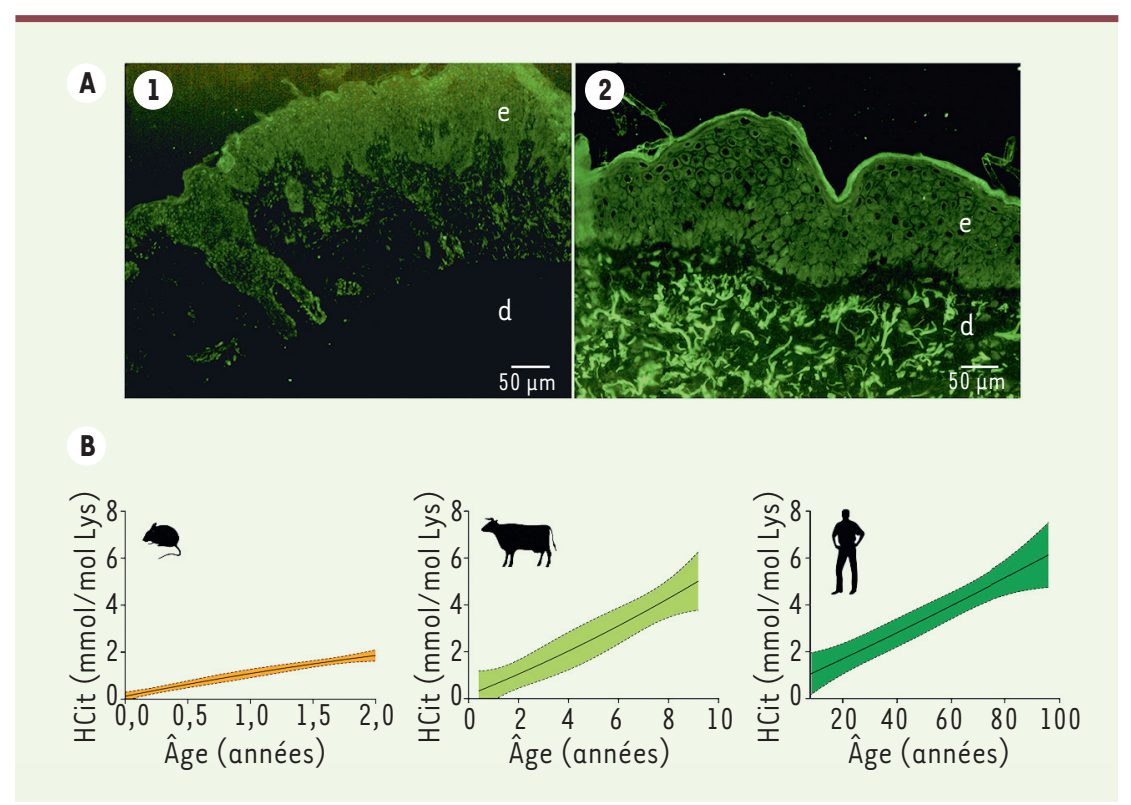

Figure 2. Accumulation des produits de carbamylation tissulaires au cours du vieillissement. A. Immuno-marquage de l'homocitrulline (HCit, en vert) sur des coupes de peau humaine jeune ( 5 mois, $\boldsymbol{A} 1$ ) et âgée (90 ans, A2) (d : derme; e : épiderme). B. Accumulation cutanée de l'HCit avec l'âge dans les trois espèces étudiées.

leure connaissance de l'implication des modifications des protéines au cours du vieillissement devrait permettre le développement de stratégies permettant de contrer le développement de telles réactions et leurs effets délétères sur l'organisme.

La carbamylation des protéines apparaît donc comme un mécanisme majeur d'altération des protéines intervenant non seulement au cours de différentes pathologies, mais également lors du vieillissement chronologique dans différentes espèces de mammifères. Nos travaux renforcent ainsi l'idée qu'aucun mécanisme biologique du vivant ne peut être correctement appréhendé si l'on ne prend en compte ces MPTNE qui affectent les protéines pendant leur séjour dans l'organisme, et qui modifient profondément leurs fonctions biologiques et leurs relations avec les cellules. $\diamond$

Protein carbamylation: when protein and chronologic ageings meet

\section{LIENS D'INTÉRÊT}

Les auteurs déclarent n'avoir aucun lien d'intérêt concernant les données publiées dans cet article.

\section{RÉFÉRENCES}

1. Gillery P, Jaisson S. Usefulness of non-enzymatic post-translational modifications-derived products (PTMDPs) as biomarkers of chronic diseases. J Proteomics 2013; $92:$ 228-39.

2. Gorisse L, Pietrement C, Vuiblet V, et al. Protein carbamylation is a hallmark of aging. Proc Natl Acad Sci USA 2016; 113 : 1191-6.

3. Maillard LC. Action des acides aminés sur les sucres; formation des mélanoïdes par voie méthodique. $C R$ Acad Sci Paris 1912 ; 154 : 66-8.

4. Sell DR, Lane MA, Johnson WA, et al. Longevity and the genetic determination of collagen glycoxidation kinetics in mammalian senescence Proc Natl Acad Sci USA 1996 ; $93:$ 485-90.

5. Jaisson S, Pietrement C, Gillery P. Carbamylationderived products (CDPs) : bioactive compounds and potential markers in chronic renal failure and atherosclerosis. Clin Chem 2011 ; 57 : 1499-505.

6. Nicholson DH, Harkness DR, Benson WE, Perterson CM. Cyanate-induced cataracts in patients with sicklecell hemoglobinopathies. Arch Ophthalmol 1976 ; 94 : 927-30.

7. Pietrement C, Gorisse L, Jaisson S, Gillery P. Chronic increase of urea leads to carbamylated proteins accumulating in tissues in a mouse model of CKD. PLoS One 2013 ; 8 : e82506.

8. Berg AH, Drechsler C, Wenger $G$, et al. Carbamylation of serum albumin as a risk factor for mortality in patients with kidney failure. Sci Transl Med 2013 ; 5 : 175 ra29.

9. Garnotel R, Sabbah N, Jaisson S, Gillery P. Enhanced activation of and increased production of matrix metalloproteinase -9 by human blood monocytes upon adhering to carbamylated collagen. FEBS Lett 2004 ; $563: 13-6$.

10. Jaisson $S$, Delevallée-Forte $C$, Touré $F$, et al. Carbamylated albumin is a potent inhibitor of polymorphonuclear neutrophil respiratory burst. FEBS Lett 2007 ; 581 : 1509-13.

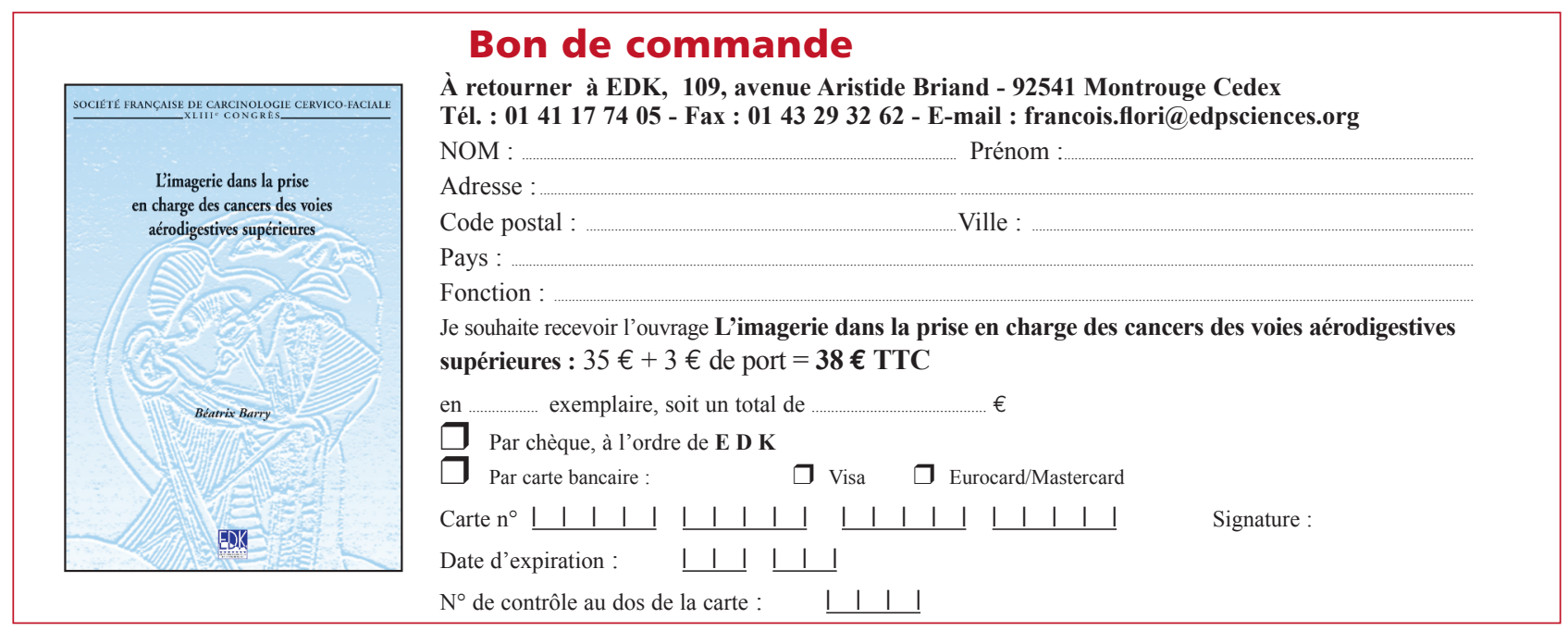

